\title{
Efecto de las lesiones condrales asintomáticas en la recuperación del paciente laboral tras una lesión traumática de rodilla
}

\author{
Effect of asymptomatic chodral on the recovery of the working \\ patient after a traumatic injury of the knee \\ Jordà-Gómez P,* Ferràs-Tarragó J,* Part-Soriano J, ${ }^{\ddagger}$ Sánchez-Alepuz E E,§ \\ Hospital IMED Valencia.
}

RESUMEN. El objetivo principal del estudio fue analizar la asociación entre la presencia de lesiones cartilaginosas asintomáticas en pacientes con lesiones traumáticas de rodilla y su tiempo de baja y gasto mutual. Los objetivos secundarios fueron describir la prevalencia y epidemiología de estas lesiones y analizar si se asocian con la presencia de obesidad y un peor resultado final. Material y métodos: Revisión retrospectiva de una cohorte de todos los pacientes a los que se les realizó una artroscopía de rodilla tras una lesión traumática en un centro mutual en el año 2018. Se recogieron datos demográficos, diagnóstico, patología condral concomitante, tratamiento, clínica al alta, tiempo total de baja y el gasto derivado de ésta. Resultados: Fueron analizados 123 pacientes con una media de edad de 47 años, no se encontraron diferencias entre sexos ni respecto a la obesidad entre los grupos con y sin lesión condral. Las lesiones cartilaginosas fueron diagnosticadas en más de un tercio de los pacientes valorados (35.25\%), la mayoría entre 48 y 53 años. La presencia de patología condral no fue un condicionante que aumentara los días de baja o el gasto total ( $p>0.05)$. En pacientes con meniscectomía, la lesión condral aumenta el tiempo de baja $(\mathrm{p}=0.03)$. Los tratamientos enfocados en la lesión condral no produjeron diferencias en cuanto a la duración de la baja ni en el gasto. Conclusión:
ABSTRACT. The objective of this study was to investigate the prevalence and epidemiology of knee cartilage lesions in the work environment, and to assess whether they increase the patient's work leave and thus also cost. We also analyzed the prevalence of concomitant pathology and how it affected recovery and final outcome. Material and methods: Monocentric retrospective cohort of patients with occupational injuries who underwent knee arthroscopy during 2018. Demographic data, diagnosis, concomitant chondral pathology, treatment, symptoms and signs at discharge, work leave and total cost were collected. Results: 123 patients were analyzed, with a mean age of 47 years. No differences were found between sexes or with respect to obesity. Asymptomatic chondral lesions were found in $35.25 \%$ of the patients, primarily the older ones (48-53 years). The presence of cartilaginous pathology did not increase days of work leave or total cost ( $p>0.05)$. In patients with meniscopathy in whom meniscectomy is performed, the chondral lesion increased the number of days of work leave $(p=0.03)$. There were no differences in the number of days of work leave nor total cost for different treatments of chondral pathology. Conclusion: The management of a concomitant chondral knee lesion is still controversial. These lesions might convey poorer functional

\section{Nivel de evidencia: IV}

\footnotetext{
* Hospital Universitari i Politècnic La Fe.

‡ Unión de Mutuas.

$\S$ Hospital IMED Valencia.
}

España.

Correspondencia:

Dr. Pablo Jordà-Gómez

Secretaría de Servicio de Ortopedia y Traumatología, Planta 6º E. Avinguda de Fernando Abril Martorell, No 106, 46026 València. E-mail: jorda.gomez.pablo@gmail.com

Recibido: 10-07-2020. Aceptado: 15-07-2021.

Citar como: Jordà-Gómez P, Ferràs-Tarragó J, Part-Soriano J, Sánchez-Alepuz E. Efecto de las lesiones condrales asintomáticas en la recuperación del paciente laboral tras una lesión traumática de rodilla. Acta Ortop Mex. 2021; 35(3): 261-265. https://dx.doi.org/10.35366/102364

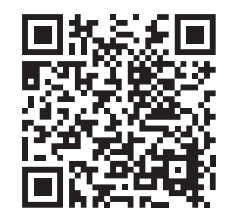


El manejo de una lesión condral concomitante a nivel de la rodilla sigue presentando controversia. Podría implicar un factor de mal pronóstico de recuperación en pacientes con meniscopatía y las terapias actuales no han mostrado un beneficio claro en estos pacientes del entorno laboral.

Palabras clave: Rodilla, artroscopía, lesión cartílago, traumática, lesión laboral.

\section{Introducción}

Las meniscopatías y la patología del ligamento cruzado anterior son dos de las lesiones más prevalentes en pacientes del mundo laboral, es decir, en individuos activos de mediana edad. Las lesiones meniscales representan alrededor de $15 \%$ de la patología de la rodilla, incidencia que aumenta cuando hay también una lesión del ligamento cruzado anterior, llegando al rango de 22 a $85 \%{ }^{1}$

La patología del cartílago articular también es frecuente en este grupo poblacional. Diversos estudios basados en artroscopías de rodilla reportan una prevalencia de alrededor de $65 \%$ en pacientes de mediana edad. ${ }^{2}$ Además, tan sólo un tercio de los casos suele aparecer una lesión del cartílago de forma aislada, siendo más común que se asocie a lesiones del menisco medial (alrededor de $40 \%$ ) y a lesiones del ligamento cruzado anterior (36\%). ${ }^{3}$

El manejo de estos pacientes en el entorno laboral presenta ciertos condicionantes, ya que es de vital importancia una pronta recuperación con la mejor funcionalidad posible del paciente para que se reincorpore a su puesto de trabajo.

Hay que tener en cuenta que pueden existir lesiones condrales focales en pacientes de edad laboral que se presenten de forma asintomática. ${ }^{4}$ La existencia de este tipo de lesiones al tratar una lesión meniscal o ligamentaria de rodilla, podría condicionar una rehabilitación más prolongada y una demora en la recuperación del paciente. ${ }^{5}$

El objetivo principal de este estudio fue analizar la asociación entre la presencia de lesiones cartilaginosas de rodilla asitomáticas en pacientes con lesiones traumáticas laborales y el tiempo de baja y gasto mutual de estos pacientes. Los objetivos secundarios fueron describir la prevalencia y epidemiología de estas lesiones y analizar si se asocian con la presencia de obesidad, así como evaluar su repercusión en el resultado final y determinar si había diferencias dependiendo del tratamiento aplicado a la lesión condral. Nuestra hipótesis era que la existencia de lesiones condrales condiciona una demora en la recuperación y vuelta al trabajo del paciente.

\section{Material y métodos}

Se realizó una revisión retrospectiva de todos los pacientes de nuestro centro mutual con edad comprendida entre 16 y 67 años a quienes se les había realizado una artroscopía de rodilla en el año 2018 con diagnóstico previo de prognosis in patients with meniscopathy. Current therapies have not shown a clear benefit in work injuries.

Keywords: Knee, arthroscopy, cartilage injury, traumatic, occupational injury.

patología meniscal, ligamentosa o condral. Se excluyeron aquellos pacientes a los que se les realizó una artroscopía para tratamiento de una fractura o lesión multiligamentosa y pacientes con un seguimiento menor de tres meses.

En todos los casos se realizó un diagnóstico y manejo inicial por parte del equipo traumatológico mediante exploración física y pruebas de imagen complementarias como radiografías y resonancia magnética (RM). Se recogieron las variables demográficas de sexo, edad y la presencia de obesidad (IMC > 30), así como el mecanismo de lesión y el diagnóstico prequirúrgico: meniscopatía, lesión de ligamento cruzado anterior (LCA), lesión condral (diagnosticada previamente por RM) o lesión combinada (patología meniscal y ligamentosa).

Se incluyó también el procedimiento artroscópico para la patología meniscal o ligamentosa, la existencia de lesión condral, el grado de lesión de la misma mediante la escala ICRS $^{6}$ y el procedimiento quirúrgico aplicado a la patología condral. Se recogió la localización de las lesiones en la superficie articular patelar, tróclea, cóndilo femoral medial y lateral y a nivel de la meseta tibial. La superficie afectada fue estimada mediante el uso del palpador artroscópico. Cuando se diagnosticaba una lesión condral, se trataba de forma expectante, con un desbridamiento, con microperforaciones o con microperforaciones e infiltración de ácido hialurónico a criterio del cirujano.

Finalmente, el paciente era seguido en consultas externas por el traumatólogo y el médico rehabilitador. Se recogió la clínica que presentaba en el momento del alta laboral y el número de días de baja que había ocasionado el proceso. El departamento económico del centro proporcionó los datos relevantes al coste total del período de baja del paciente así como su profesión.

Todos los análisis estadísticos fueron realizados utilizando el software libre R Commander 3.4.3. Se analizó la normalidad de la distribución de la variable edad mediante los test de Shapiro-Wilk y de Lilliefors (Kolmogorov-Smirnov). El estudio analítico de los parámetros cualitativos se realizó mediante el test $\chi^{2}$ y para los datos cuantitativos se utilizó el t-Student. Los resultados arrojados por los test estadísticos se consideraron significativos cuando la $\mathrm{p}<0.05$.

\section{Resultados}

Se identificaron 123 pacientes, con edad media de 47 años (IC 95\% 45.75-49.69 p valor <2.2e-16). La edad pre- 
sentó una distribución normal ( $\mathrm{p}>0.05)$. El diagnóstico más frecuente fue el de lesión meniscal (82 pacientes, 67.2\%), seguido de lesiones combinadas (32 pacientes, 26.2\%) y lesiones del LCA (ocho pacientes, 6.5\%). La causa de la lesión fue en todos los casos un evento traumático.

Los pacientes con lesiones condrales presentaron una edad media significativamente mayor (49 años vs 42 años sin lesión condral, $\mathrm{p}<0.05)$. Las lesiones condrales asintomáticas fueron más frecuentes en pacientes de edad comprendida entre 48 y 53 años (con un IC 95\% entre 48.753.5 años, con un p-valor $<0.05$ ). No hubo diferencias en la prevalencia de lesiones condrales entre sexos $(p=0.58) \mathrm{ni}$ entre pacientes obesos vs no-obesos $(\mathrm{p}=0.54)$, si bien hubo una baja prevalencia de obesidad (cinco pacientes, $0.04 \%$ ). La distribución de las lesiones condrales encontradas en cada grupo de diagnóstico fue homogénea, sin detectarse diferencias significativas entre ellos $(\mathrm{p}=0.08)$ (Tabla 1).

Las lesiones cartilaginosas fueron diagnosticadas en más de un tercio de los pacientes valorados (35.25\%). De éstas, $67.3 \%$ afectaron al cóndilo femoral medial y $18.5 \%$ a la rótula, siendo en su mayoría grado II-III de la ICRS (Tabla 2). Destaca también que las lesiones condrales a nivel de la meseta tibial y de la región troclear siempre fueron concomitantes con otras lesiones o a nivel condilar o a nivel rotuliano.

Al comparar la existencia de lesiones condrales con respecto a los días de baja totales, se observa que los pacientes con el cartílago afectado presentan mayor duración (154 días), respecto a los no afectados (125 días), sin hallarse diferencias estadísticamente significativas $(\mathrm{p}=0.11)$. Lo mismo ocurre con el gasto, donde los pacientes con lesión presentaron un gasto total de 4,712.49€ respecto a los $4,399.66 €$ de los no afectados, de nuevo sin significancia estadística $(\mathrm{p}=0.61)$.

En todos los casos de lesión condral se realizó algún tipo de actitud terapéutica. El desbridamiento de la zona afectada se realizó en $69.7 \%$, las perforaciones en $23.3 \%$ y la infiltración con ácido hialurónico tras perforaciones en $6.9 \%$. No hubo diferencias significativas en cuanto a la duración de la baja $(\operatorname{Pr}(F)=0.21)$ entre los tres tratamientos (Figura 1). No se pudo estratificar por gravedad de la lesión condral por falta de potencia estadística. Tampoco observamos diferencias de significación en el gasto total del tratamiento en función de la terapéutica elegida para la lesión condral $(\operatorname{Pr}(F)=0.6)$. La lesión condral sí aumentó

\begin{tabular}{|c|c|c|}
\hline \multicolumn{3}{|c|}{$\begin{array}{l}\text { Tabla 1: Lesiones condrales diagnosticadas } \\
\text { respecto al tipo de lesión del paciente. }\end{array}$} \\
\hline \multirow[b]{2}{*}{ Diagnóstico } & \multicolumn{2}{|c|}{ Lesión del cartílago, n } \\
\hline & No & Sí \\
\hline Ligamento cruzado anterior & 8 & 0 \\
\hline Lesión combinada & 19 & 13 \\
\hline Meniscopatía & 52 & 30 \\
\hline
\end{tabular}

Tabla 2: Tipos de lesiones condrales.

$\begin{array}{lc}\text { Lesión condral (condropatía) } & \text { n (\%) } \\ \text { Grado III CFM } & 15(34.8) \\ \text { Grado II CFM } & 14(32.5) \\ \text { Grado III rotuliana } & 5(11.6) \\ \text { Grado IV CFM } & 4(9.3) \\ \text { Grado III meseta tibial (concomitante) } & 4(9.3) \\ \text { Grado II rotuliana } & 3(6.9) \\ \text { Grado II troclear (concomitante) } & 3(6.9) \\ \text { Grado II CFE } & 1(2.3) \\ \text { Grado III CFE } & 1(2.3)\end{array}$

Las lesiones de meseta tibial y de la zona troclear fueron en todos los casos concomitantes a otras lesiones condrales.

CFM = cóndilo femoral medial, $\mathrm{CFE}$ = cóndilo femoral externo.

el tiempo de baja significativamente en aquellos meniscópatas en los que se realizó meniscectomía ( $\mathrm{p}=0.03$, IC 95\% 82-4) (Figura 2).

Al valorar la clínica del paciente al alta de forma general, no se observaron diferencias significativas entre el grupo con lesión cartilaginosa y el grupo sin ella, pero se vio una tendencia a un mayor riesgo de clínica residual en el grupo de lesión condral diagnosticada $(\mathrm{p}=0.06)$.

\section{Discusión}

Las lesiones en pacientes del entorno laboral, al igual que los atletas ${ }^{7,8,9}$ o profesionales militares, ${ }^{10,11}$ presentan fuertes condicionantes a la hora de plantear cuál es el tratamiento más idóneo, ya que van a estar muy influenciadas por factores internos y externos al paciente. Ahí reside la importancia de intentar detectar e individualizar cada uno de estos factores para poder tratar con éxito este tipo de patología y conseguir una rápida recuperación del paciente.

Las lesiones meniscales o del LCA es muy común que asocien algún tipo de lesión cartilaginosa ${ }^{12,13,14}$ que podría afectar la rehabilitación posterior y una precoz vuelta al entorno laboral. ${ }^{12}$ En nuestro estudio los defectos en el cartílago fueron hallados en más de un tercio de los pacientes, un porcentaje algo menor que el aproximado $50 \%$ de los casos reportados en la literatura. ${ }^{2,3,15,16}$

Ni la presencia ni la gravedad de las lesiones condrales se asoció con un aumento en el gasto ni el tiempo de baja en nuestra cohorte. Este resultado no se corresponde con nuestra percepción de la práctica clínica y lo atribuimos a que:

1. Los pacientes de nuestra cohorte habían sido remitidos por dolor tras un traumatismo en un contexto laboral de forma aguda, no por sospecha de patología degenerativa, por lo que se consideraron estas lesiones condrales como hallazgo casual en contexto de otra lesión.

2. La edad de nuestra población fue joven en relación a la edad media de inicio de síntomas artrósicos.

3. Si se estratifica por el grado de lesión condral, nuestra cohorte carece de potencia estadística suficiente para 
encontrar diferencias en el tiempo de baja o el gasto en el caso de que las hubiera.

Así, dado que la lesión condral no fue la causa de la baja, es concebible que no sea un condicionante primario para la recuperación de este tipo de pacientes específicamente. No obstante, también es posible que la presencia de una lesión condral en el contexto de una lesión laboral sintomática (LCA, meniscopatía o lesión combinada) sí condicione el pronóstico de esta lesión laboral como se ha reportado previamente. ${ }^{13,14}$ En especial, en casos de lesión meniscal: una publicación sobre meniscopatías en el entorno laboral hizo referencia a una posible relación de los defectos osteocondrales con las lesiones meniscales; ${ }^{16}$ otro grupo, al observar factores que predisponían a artroplastía de rodilla tras artroscopía, se destacó la meniscectomía y las lesiones condrales mayores de grado II. ${ }^{17}$ Nuestra cohorte sí apoya estos datos, pues al observar al grupo de pacientes que recibió meniscectomía, objetivamos un aumento significativo en el tiempo de baja en aquéllos que tenían lesiones condrales.

Para el manejo de las lesiones condrales se utilizaron tres diferentes actitudes terapéuticas (desbridamiento/hialurónico/microperforaciones), ya valoradas en otros estudios. ${ }^{18,19,20,21}$ No observamos diferencias significativas en cuanto a la duración de la baja. Un sesgo destacable en este análisis sería el probable factor de confusión (gravedad de la lesión condral), por el que no se pudo corregir por falta de potencia en el análisis estratificado. En cuanto al gasto, tampoco se observaron diferencias, posiblemente por una mayor relación del gasto con el tratamiento de la lesión primaria traumática y no por la lesión condral. La posibilidad de realizar terapias regenerativas en este tipo de lesiones podría favorecer su recuperación, tal como indican algunos estudios. ${ }^{22}$

Uno de los objetivos secundarios era observar si la obesidad podía ser otro factor que afectara la recuperación, sin que obtuviéramos resultados concluyentes. Atribuimos este resultado a que si bien la obesidad predispone a la artrosis y las lesiones condrales, lo hace habitualmente después de

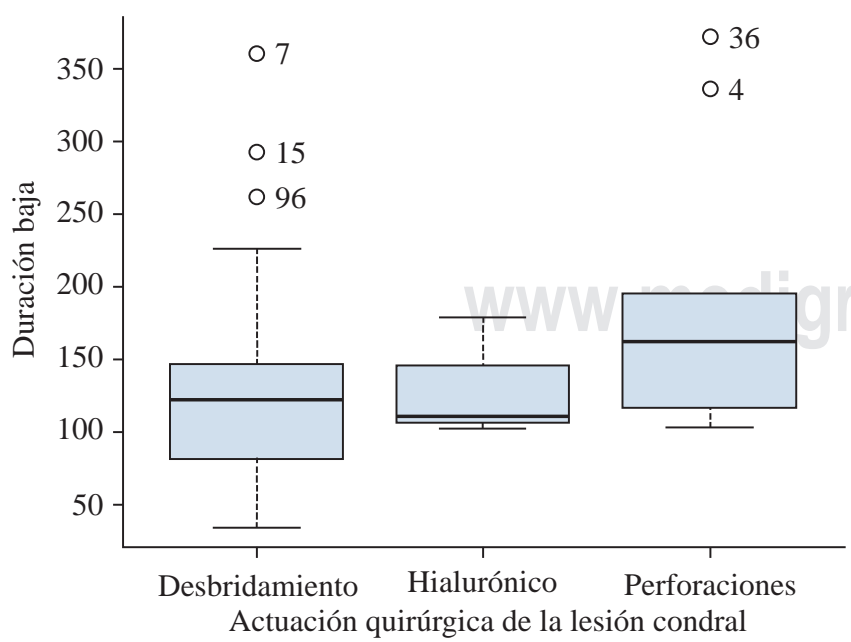

Figura 1: Duración de la baja en pacientes con lesión condral en función del tratamiento administrado.

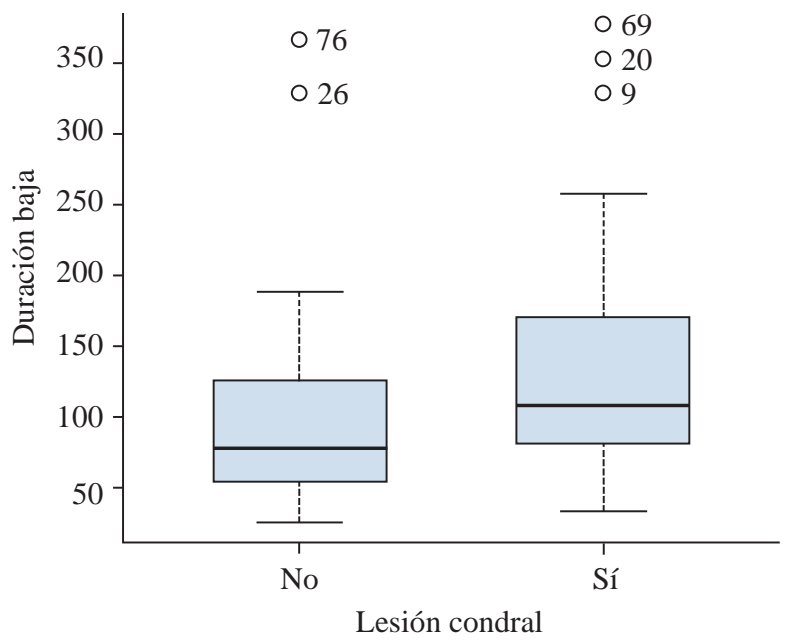

Figura 2: Duración de la baja en función de la presencia o no de la lesión condral en pacientes con lesión meniscal en los que se realiza una meniscectomía.

una media de edad de 47 años de nuestra cohorte. ${ }^{23}$ Así, es improbable que en el grupo poblacional estudiado la obesidad hubiera llegado a producir lesiones condrales. Adicionalmente, la prevalencia de obesidad en nuestra cohorte fue muy baja. Por todo ello, aunque la relación de la obesidad con las lesiones condrales es conocida, nuestro estudio careció de potencia como para que dicha relación se viese reflejada en nuestros resultados.

Entre las limitaciones de esta investigación se incluyen su carácter retrospectivo y la existencia de múltiples factores confusores exógenos, por ejemplo, la colaboración y voluntad de reincorporación laboral. Entre las fortalezas destaca una fuerte validez interna gracias a que fue un mismo equipo quirúrgico el que trató a todos y un protocolo intenso y homogéneo de rehabilitación.

\section{Conclusión}

La existencia de una lesión condral concomitante a nivel de la rodilla es una patología de manejo difícil y controvertido. Podría ser un factor de mal pronóstico funcional en pacientes con meniscopatía. Las terapias actuales no han mostrado un beneficio claro en estos pacientes del entorno laboral. Un nuevo enfoque con terapias más dirigidas a una recuperación de la lesión tiene el potencial de ofrecer resultados más satisfactorios.

\section{Referencias}

1. O'Connor DP, Laughlin MS, Woods GW. Factors related to additional knee injuries after anterior cruciate ligament injury. Arthrosc J Arthrosc Relat Surg Off Publ Arthrosc Assoc N Am Int Arthrosc Assoc. 2005; 21(4): 431-8. doi: 10.1016/j. arthro.2004.12.004.

2. Aroen A, Loken S, Heir S, et al. Articular cartilage lesions in 993 consecutive knee arthroscopies. Am J Sports Med. 2004; 32(1): 211-5. doi: 10.1177/0363546503259345. 
3. Widuchowski W, Widuchowski J, Trzaska T. Articular cartilage defects: study of 25,124 knee arthroscopies. Knee. 2007; 14(3): 17782. doi: 10.1016/j.knee.2007.02.001.

4. Flanigan DC, Harris JD, Trinh TQ, Siston RA, Brophy RH. Prevalence of chondral defects in athletes' knees: a systematic review. Med Sci Sports Exerc. 2010; 42(10): 1795-801. doi: 10.1249/ MSS.0b013e3181d9eea0.

5. Bedi A, Feeley BT, Williams RJ. Management of articular cartilage defects of the knee. J Bone Joint Surg Am. 2010; 92(4): 994-1009. doi: 10.2106/JBJS.I.00895.

6. Smith GD, Taylor J, Almqvist KF, et al. Arthroscopic assessment of cartilage repair: a validation study of 2 scoring systems. Arthroscopy. 2005; 21(12): 1462-7. doi: 10.1016/j.arthro.2005.09.007.

7. Baylis WJ, Rzonca EC. Common sports injuries to the knee. Clin Podiatr Med Surg. 1988; 5(3): 571-89.

8. Drez D Jr. Arthroscopic evaluation of the injured athlete's knee. Clin Sports Med. 1985; 4(2): 275-8.

9. Adirim TA, Cheng TL. Overview of injuries in the young athlete. Sports Med. 2003; 33(1): 75-81. doi: 10.2165/00007256-20033301000006.

10. Szuflita NS, Neal CJ, Rosner MK, Frankowski RF, Grossman RG. Spine injuries sustained by U.S. military personnel in combat are different from non-combat spine injuries. Mil Med. 2016; 181(10): 1314-23. doi: 10.7205/MILMED-D-15-00332.

11. Schoenfeld AJ, Dunn JC, Belmont PJ. Pelvic, spinal and extremity wounds among combat-specific personnel serving in Iraq and Afghanistan (2003-2011): a new paradigm in military musculoskeletal medicine. Injury. 2013; 44(12): 1866-70. doi: 10.1016/j. injury.2013.08.001.

12. Murrell GAC, Maddali S, Horovitz L, Oakley SP, Warren RF. The effects of time course after anterior cruciate ligament injury in correlation with meniscal and cartilage loss. Am J Sports Med. 2001; 29(1): 9-14. doi: 10.1177/03635465010290012001.

13. Lewandrowski KU, Müller J, Schollmeier G. Concomitant meniscal and articular cartilage lesions in the femorotibial joint. Am J Sports Med. 1997; 25(4): 486-94. doi: 10.1177/036354659702500411.

14. Codesido P, Leyes M, Forriol F. Relación entre el mecanismo de producción y las lesiones concomitantes en las roturas del ligamento cruzado anterior. Rev Esp Cir Ortop Traumatol. 2009; 53(4): 231-6. doi: 10.1016/j.recot.2009.02.001.
15. Curl WW, Krome J, Gordon ES, Rushing J, Smith BP, Poehling GG. Cartilage injuries: a review of 31,516 knee arthroscopies. Arthrosc J Arthrosc Relat Surg. 1997; 13(4): 456-60. doi: 10.1016/S07498063(97)90124-9.

16. Alves S, Guerra PF, Almeida JF, Ramos V, Goncalves S, Beja da Costa P. Resultados clínicos a corto plazo de la sutura meniscal en pacientes asociados a seguros laborales. Rev Esp Artrosc Cir Articul. 2014; 21(2): 101-8. doi: 10.1016/j.reaca.2015.01.002.

17. Figueroa D, Calvo R, Villalón I, Tuca MJ, Vaisman A, Valdés M. Factores clínicos y hallazgos en la artroscopía de pacientes con artrosis de rodilla que favorecen la conversión a artroplastía total. Rev Esp Cir Ortop Traumatol. 2013; 57(4): 263-7. doi: 10.1016/j.recot.2013.04.003.

18. Figueroa D, Espinosa M, Calvo R, et al. Tratamiento de lesiones condrales agudas de espesor completo con ácido hialurónico de alto peso molecular; un modelo experimental. Rev Esp Cir Ortop Traumatol. 2014; 58(5): 261-6. doi: 10.1016/j.recot.2014.03.001.

19. Case JM, Scopp JM. Treatment of articular cartilage defects of the knee with microfracture and enhanced microfracture techniques. Sports Med Arthrosc Rev. 2016; 24(2): 63-8. doi: 10.1097/ JSA.0000000000000113.

20. Behery O, Siston RA, Harris JD, Flanigan DC. Treatment of cartilage defects of the knee: expanding on the existing algorithm. Clin J Sport Med. 2014; 24(1): 21-30. doi: 10.1097/JSM.0000000000000004.

21. Devitt BM, Bell SW, Webster KE, Feller JA, Whitehead TS. Surgical treatments of cartilage defects of the knee: systematic review of randomised controlled trials. Knee. 2017; 24(3): 508-17. doi: 10.1016/j.knee.2016.12.002.

22. Ahmed TAE, Hincke MT. Strategies for articular cartilage lesion repair and functional restoration. Tissue Eng Part B Rev. 2010; 16(3): 305-29. doi: 10.1089/ten.TEB.2009.0590.

23. Haviv B, Bronak S, Thein R. Correlation between body mass index and chondral lesions in isolated medial meniscus tears. Indian $J$ Orthop. 2015; 49(2): 176-80. doi: 10.4103/0019-5413.152456.

Financiamiento: Los autores declaran no tener ningún tipo de financiación externa a la de la institución principal.

Conflicto de intereses: Los autores declaran no tener ningún conflicto de intereses. 